\title{
Correction to: Cardiovascular Complications in Patients with COVID-19: Consequences of Viral Toxicities and Host Immune Response
}

\author{
Han Zhu ${ }^{1,2,3} \cdot$ June-Wha Rhee ${ }^{1,2,3} \cdot$ Paul Cheng ${ }^{1,2,3} \cdot$ Sarah Waliany $^{1} \cdot$ Amy Chang $^{1,4} \cdot$ Ronald M. Witteles $^{1,3}$. \\ Holden Maecker ${ }^{5,6} \cdot$ Mark M. Davis $^{5,6,7}$. Patricia K. Nguyen ${ }^{1,2,3} \cdot$ Sean M. Wu ${ }^{1,2}$
}

Published online: 13 May 2020

(C) Springer Science+Business Media, LLC, part of Springer Nature 2020

\section{Correction to: Current Cardiology Reports (2020) 22:32. https://doi.org/10.1007/s11886-020-01292-3}

It has been pointed out that the second paragraph of the section "Treatments for SARS-CoV-2 Infection" contains an error. The original article has been corrected.

Publisher's Note Springer Nature remains neutral with regard to jurisdictional claims in published maps and institutional affiliations.

The online version of the original article can be found at https://doi.org/ 10.1007/s11886-020-01292-3

Sean M. Wu

smwu@stanford.edu

Han Zhu

hanzhu@ stanford.edu

June-Wha Rhee

jwrhee@stanford.edu

Paul Cheng

chengpa@stanford.edu

Sarah Waliany

swaliany@stanford.edu

Amy Chang

axc421@stanford.edu

Ronald M. Witteles

witteles@stanford.edu

Holden Maecker

maecker@stanford.edu
Mark M. Davis

mmdavis@stanford.edu

Patricia K. Nguyen

pknguyen@stanford.edu

1 Department of Medicine, Stanford University, Room G1120A, Lokey Stem Cell Building, 265 Campus Drive, Stanford, CA 94305, USA

2 Stanford Cardiovascular Institute, Stanford, CA, USA

3 Division of Cardiovascular Medicine, Stanford University, Stanford, CA, USA

4 Division of Infectious Disease, Stanford University, Stanford, CA, USA

5 Department of Microbiology and Immunology, Stanford University, Stanford, CA, USA

6 Stanford Institute for Immunity, Transplantation and Infection, Stanford University School of Medicine, Stanford, CA, USA

7 Howard Hughes Medical Institute, Stanford, CA, USA 